\title{
43. A Problem Concerning the Second Fundamental Theorem of Lie.
}

\author{
By Kôsaku Yosida. \\ Mathematical Institute, Faculty of Science, Osaka Imperial University. \\ (Comm. by T. YosiE, M.I.A., May 12, 1937.)
}

§1. The problem and the theorem.

Let $\Re$ denote the set of all the matrices of a fixed degree, say $n$, with complex numbers as coefficients. We introduce a topology in $\Re$ by the absolute value

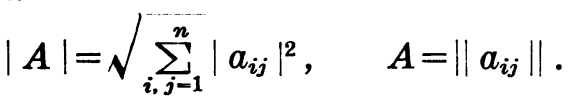

If $\mathbb{B}$, a subset of non-singular matrices $\in \Re$, is a group with respect to the matrix-multiplication, it is a topological group by the distance $|A-B|$.

The topological group $\mathbb{B}$ is called a Lie group, if there exist a finite number, say $m$, of elements $X_{1}, X_{2}, \ldots \ldots, X_{m} \in \mathfrak{R}$ which satisfy the conditions :

1). $X_{1}, X_{2}, \ldots \ldots, X_{m}$ are linearly independent with real coefficients.

2). $\exp \left(\sum_{i=1}^{m} t_{i} X_{i}\right) \in \mathbb{E S}, t$ real. ${ }^{1)}$

3). There exists a positive $\varepsilon$ such that any element $A \in \mathbb{B}$ may be represented uniquely in the form

$$
A=\exp \left(\sum_{i=1}^{m} t_{i} X_{i}\right), t \text { real, }
$$

if $|A-E| \leqq \varepsilon$ ( $E$ the unit-matrix of $\Re)$.

By a theorem of $\mathrm{J}$. von Neumann ${ }^{2}$ (S) is a Lie group if and only if it is locally compact. Here, for convention, a discrete group is also called a Lie group. If $\mathbb{B}$ is a Lie group, the set $\mathfrak{F}$ of all the elements $\sum_{i=1}^{m} t_{i} X_{i}, t$ real, satisfies :

$(\alpha)$. $\Im$ is a real linear space which has a finite base with real coefficients, viz, $X_{1}, X_{2}, \ldots \ldots, X_{m}$.

(B). $[X, Y]=X Y-Y X \in \mathfrak{\Im}$ with $X, Y \in \mathfrak{\Im}$.

$\Im$ is called the Lie ring of the Lie group $\mathbb{B}$, the two ring-operations being the vector-addition and the commutator-multiplication $[X, Y]$. It is the set of all the differential quotients of $(S)$ at $E^{3)}$ The differential quotient of $\mathbb{S}$ at $E$ is defined by $\lim _{i \rightarrow \infty}\left(\left(A_{i}-E\right) / \varepsilon_{i}\right)$, where $A_{i}(\neq E) \in \mathbb{S}$ and real $\varepsilon_{i}(\neq 0)$ are such that $\lim _{i \rightarrow \infty} A_{i}=E, \lim _{i \rightarrow \infty} \varepsilon_{i}=0$.

1) $\exp (X)=\sum_{n=0}^{\infty}\left(X^{n} / n !\right)$

2) See K. Yosida: Jap. J. of Math. 13 (1936), p. 7. Neumann's original statement (M. Z. 30 (1929), p. 3) reads as follows :

$B$ is a Lie group if $B$ is closed in the group of all the non-singular matrices $\in \mathfrak{R}$.

3) Cf. K. Yosida: loc. cit. 
Conversely let $\bar{\Im}$ denote a subset of $\Re$ which satisfies $(\alpha)$ and $(\beta)$. Then, by the second fundamental theorem of Lie, the set $\overline{\mathfrak{S}}$ of all the elements of the form

$$
\exp \left(\sum_{i=1}^{m} t_{i} X_{i}\right), t \text { real and } \sum_{i=1}^{m}\left|t_{i}\right|<\varepsilon, \quad \varepsilon>0,
$$

constitutes a Lie group-germ. That is, if $X, Y \in \overline{\mathbb{S}}$ are sufficiently near $E, X^{-1}$ and $Y X$ also $\in \overline{\mathfrak{G}}$. $\bar{\Im}$ is called the Lie ring of the Lie group-germ $\overline{\text { S. }}$.

Then the set $\widetilde{S}$ of all the products of a finite number of elements $\in \overline{(S)}$ and of the limit matrices of such products, so long as they are non-singular, forms a locally compact group. Hence $\widetilde{\mathbb{S}}$ is a Lie group. Let $\widetilde{\mathfrak{J}}$ be the Lie ring of this Lie group $\widetilde{\mathfrak{S}}$, then $\widetilde{\mathfrak{J}} \supseteqq \widetilde{\Im}$. However, $\widetilde{\Im}$ does not necessarily coincide with $\widetilde{\mathfrak{J}}$, as the following example shows us :

$$
\text { the base of } \bar{\Im}=\left\|\begin{array}{cc}
\sqrt{-1} & 0 \\
0 & \tau \sqrt{-1}
\end{array}\right\|, \tau / 2 \pi \text { irrational. }
$$

Hence the Lie group-germ $\overline{\mathbb{S}}$ is not necessarily a vicinity of the identity of the topological group ().

Thus it may be of some interest to obtain the conditions by which $\bar{\Im}$ coincides with $\widetilde{\Im}$. As an answer to this problem, I intend to prove the following

Theorem. The Lie group-germ $\overline{\mathbb{G}}$ is a vicinity of the identity of the Lie group $\widetilde{\mathbb{S}}$, if the ring $\overline{\mathfrak{F}}$ is irreducible.

Here $\overline{\mathfrak{J}}$ is called irreducible if the group $\widetilde{\mathfrak{S}}$ is irreducible, that is, if all the matrices of $\bar{\Im}$ are not simultaneously similar to the matrices of the form

$$
\left\|\begin{array}{cc}
A & 0 \\
* & B
\end{array}\right\|
$$

$\S 2$. The proof of the theorem.

Lemma 1. $\overline{\mathbb{S}}$ is a Lie invariant subgroup-germ of $\widetilde{\mathbb{S}}$, viz. $B A B^{-1} \in \overline{\mathcal{G}}$ for any $B \in \widetilde{\mathcal{G}}$ if $A \in \overline{\mathbb{G}}$ is sufficiently near $E$.

Proof. Let $A=\exp (X), X \in \bar{\Im}$. Then $B A B^{-1}=\exp \left(B X B^{-1}\right)$ and $B X B^{-1}$ tends to 0 as $X$ tends to 0 . Thus it is sufficient to prove

$$
B X B^{-1} \in \bar{\Im} \text { with } X \in \bar{\Im}, \quad \text { if } B \in \widetilde{\mathbb{S}} \text {. }
$$

$\left.{ }^{*}\right)$ is evident in the special case $B \in \overline{\mathbb{G}}$, for then the transformation $X \rightarrow B X B^{-1}$ is induced by the so-called linear adjoint Lie group-germ of $\overline{\mathfrak{G}}$. The general case $B \in \widetilde{\mathbb{S}}$ may be obtained from this special case, by limiting process.

Lemma 2 (due to E. Cartan"). The vicinity of the identity of the irreducible Lie group $\widetilde{\mathbb{S}}$ is a direct product of a semi-simple Lie

1) E. Cartan: Ann. Ec. Norm. Sup. (3) 26 (1909), p. 148. For the proof see H. Freudenthal : Ann. of Math. 37, 1 (1936), p. 63. In the course of the proof of our theorem, $\bar{\Theta}_{i}(i=1,2)$ will be proved to be not only Lie group-germ but also a vicinity of the identity of the Lie group. 
group-germ $\bar{G}_{1}$ and an abelian Lie group-germ $\overline{\mathfrak{G}}_{2}$, where $\operatorname{det}(A)=1$ for any $A \in \overline{\mathfrak{S}}_{1}$ and the matrices of $\overline{\mathfrak{G}}_{2}$ are all of the form $\alpha E$, $\alpha$ denoting complex numbers.

As a special case of this Lemma we have

Lemma $2^{\prime} . \quad \widetilde{\mathfrak{S}}$ is a semi-simple Lie group if $\overline{\mathfrak{\Im}}$ is irreducible and

$$
\text { trace }(X)=0 \quad \text { for } X \in \overline{\mathfrak{\Im}} \text {. }
$$

Proof. For then the matrices of $\widetilde{\mathcal{S}}$ and hence of $\widetilde{\mathbb{S}}$ are all of determinant 1 . $^{\text {) }}$

The above condition $\left({ }^{* *}\right)$ is surely satisfied if the Lie ring $\overline{\mathfrak{J}}$ is semi-simple. For a semi-simple Lie ring $\bar{\Im}$ coincides with its commutator-ring, ${ }^{2}$ that is, any element of $\mathfrak{\Im}$ may be obtained as the commutator-product $[X, Y]$, where $X$ and $Y \in \bar{\Im}$.

Proof of the theorem. By Lemma 1 the sub-ring $\Im$ is an ideal in $\widetilde{\Im}$, viz. $[X, Y] \in \bar{\Im}$ for $X \in \bar{\Im}, Y \in \widetilde{\Im}$. We will prove that this ideal $\bar{\Im}$ is a direct summand of the Lie ring $\widetilde{\Im}$.

By Lemma 2 the Lie ring $\widetilde{\Im}$ is a direct sum of the semi-simple Lie ring $\bar{\Im}_{1}$ of the Lie group-germ $\overline{\mathfrak{S}}_{1}$ and the abelian Lie ring $\bar{\Im}_{2}$ of the Lie group-germ $\overline{\mathfrak{F}}_{2}$. Thus $\widetilde{\Im}_{1}$ is commutative with $\bar{\Im}_{2}:[X, Y]=0$ for $X \in \widetilde{\Im}_{1}, Y \in \widetilde{\Im}_{2}$.

The semi-simple Lie ring $\bar{\Im}_{1}$ is a direct sum of simple and semisimple ideals, by a theorem of E. Cartan. ${ }^{3)}$ Hence any ideal of $\mathbb{\Im}_{1}$ is semi-simple. As $\overline{\mathcal{G}}_{2}$ consists of the matrices of the form $a E$, the base of the abelian Lie ring $\bar{\Im}_{2}$ is either

i). $a E$, where $a$ denotes a real or complex number $\left(a=0\right.$ if $\left.\widetilde{\Im}_{2}=0\right)$, or

ii). $E$ and $\sqrt{-1} E$.

Thus, in any case, $\widetilde{\Im}$ is a direct sum of simple ideals. Hence the ideal $\bar{\Im}$ is a direct summand of $\widetilde{\Im}$. We next prove that $\bar{\Im} \geqq \bar{\Im}_{1}$.

Let $\widetilde{\mathfrak{J}}=\overline{\mathfrak{J}}+\widetilde{\Im}^{\prime}$ be a direct decomposition of $\widetilde{\mathfrak{J}}$. Then, as $\overline{\mathfrak{J}}$ and $\bar{\Im}^{\prime}$ are ideals in $\widetilde{\Im}, \bar{\Im}$ is commutative with $\overline{\mathfrak{I}}^{\prime}$ :

$$
\left.{ }^{* * *}\right) \quad[X, Y]=0 \quad \text { for } X \in \bar{\Im}, \quad Y \in \overparen{\Im}^{\prime} .
$$

Hence, if $\bar{\Im}$ does not contain $\overline{\Im_{1}}$, there must exist a semi-simple ideal $\widetilde{\Im}_{1}^{\prime} \leqq \widetilde{\Im}_{1}$, commutative with $\widehat{\Im}$ by $\left({ }^{* * *}\right)$. Thus the matrices $€ \widetilde{\mathbb{S}}$ of the form $\exp (X), X \in \mathfrak{J}_{1}^{\prime}$, are permutable with every matrix of the irreducible group-germ (F). Hence, by Schur's Lemma, exp $(X)\left(X \in \mathfrak{I}_{1}^{\prime}\right)$ and consequently every matrix $\epsilon \mathfrak{I}_{1}^{\prime}$ must be of the form $a E$. $\mathfrak{I}_{1}^{\prime}$ is thus an abelian Lie ring and hence is not semi-simple. This is a contradiction, and so we must have $\bar{\Im} \geqq \widetilde{\Im}_{1}$.

The same reasoning shows that, if $\bar{\Im}$ is irreducible and semisimple, we must have $\widetilde{\mathfrak{F}}=\widetilde{\mathfrak{J}}$. For, then $\widetilde{\mathfrak{J}}$ is semi-simple by Lemma 2 '. Hence, in the above Lemma $2, \overline{\mathcal{G}}_{1}$ and $\overline{\mathfrak{G}}_{2}$ are not only Lie groupgerm but also the vicinities of the identities of Lie groups.

Next we will prove that $\overline{\mathfrak{J}} \supseteqq \overline{\mathfrak{S}}_{2}$. There are two cases.

1) $\operatorname{det} .(\exp (X))=\exp (\operatorname{trace}(X))$.

2) See, for example, H. Freudenthal: loc. cit.

3) E. Cartan: Théses (1894), p. 53. 
Case 1. Base of $\bar{\Im}_{2}=a E\left(a=0\right.$ if $\left.\bar{\Im}_{2}=0\right)$.

Assume that $\widetilde{\Im}_{2} \neq 0$ and $\bar{\Im}=\bar{\Im}_{1}$. Then the group-germ $\overline{\mathfrak{G}}$ is a vicinity of the identity of the Lie group $\widetilde{\mathscr{G}}_{1}$ whose Lie ring are $\widetilde{\Im}_{1}=\widetilde{\Im}_{\mathfrak{Y}}$. Thus $\bar{\Im}_{2}=0$, contrary to the hypothesis. This proves $\bar{\Im} \supseteqq \bar{\Im}_{2}$.

Case 2. Base of $\bar{\Im}_{2}=E$ and $\sqrt{-1} E$.

If both $E$ and $\sqrt{-1} E$ do not belong to $\bar{\Im}$, we obtain $\bar{\Im}_{2}=0$ as above, contrary to the hypothesis $\widetilde{\Im}_{2} \neq 0$. Next let either one of $E$ and $\sqrt{-1 E}, E$ for example, belong to $\bar{\Im}$. Then, as $E$ is permutable with every matrix, any matrix $\in \widetilde{\mathbb{S}}$ must be of the form

$$
A_{1} A_{2} \ldots \ldots A_{k} Y, \text { where }\left\{\begin{array}{l}
A_{i} \in \text { the intersection }\left(\overline{\mathfrak{S}} \cdot \overline{\mathbb{G}_{1}}\right), \\
Y=\exp (t E), t \text { real, }
\end{array}\right.
$$

or the limit matrix of such matrices. Thus, by Lemma 2 , det. $(X)=$ $\exp (t), t$ real, for $X \in \widetilde{\mathbb{S}}$, and hence $X$ is not of the form $\exp (s \cdot \sqrt{-1} E)$, $s$ real. Then $\sqrt{-1} E$ does not belong to the Lie ring $\widetilde{\Im}$. This is a contradiction, and so we must have $\overline{\mathfrak{\Im}} \supseteqq \overline{\mathfrak{I}}_{2}$.

Thus, in any case, $\bar{\Im}=\widetilde{\Im}$.

Q. E. D. 